\title{
Development of a multiblock procedure for automated generation of two-dimensional quadrilateral meshes of gear drives
}

\author{
Victor Roda-Casanova*, Francisco Sanchez-Marin \\ Department of Mechanical Engineering and Construction, Universitat Jaume I, Castellon, Spain
}

\begin{abstract}
This article describes a new multiblock procedure for automated generation of two-dimensional quadrilateral meshes of gear drives. The typical steps of the multiblock schemes have been investigated in depth to obtain a fast and simple way to mesh planar sections of gear teeth, allowing local mesh refinement and minimizing the appearance of distorted elements in the mesh.

The proposed procedure is completed with two different mesh quality enhancement techniques. One of them is applied before the mesh is generated, and reduces the distortion of the mesh without increasing the computational time of the meshing process. The other one is applied once the mesh is generated, and reduces the distortion of the elements by means of a mesh smoothing method.

The performance of the proposed procedure has been illustrated with several numerical examples, which demonstrate its ability to mesh different gear geometries under several meshing boundary conditions.
\end{abstract}

Keywords: gear drives, finite element analysis, mesh generation

\section{Introduction}

The finite element analysis (FEA) plays an important role during the design stages of gear drives, as it allows to determine the stress state of the gears under load [1,2], to investigate the formation of the bearing contact during the transmission of power $[3,4,5]$ and to predict the temperature field of the gears in operating conditions [6,7], among other things. Performing a finite element analysis requires the development of a finite element model of the transmission, which is accomplished through three tasks [8]: (i) generation of the finite element mesh of the gear drive, (ii) definition of the contact surfaces and (iii) establishment of the loading and the boundary conditions of the gear drives.

Let us draw our attention into the first one of these tasks, in which a finite element mesh of the gear drive is generated. This task can be divided into two sequential steps: in the first step, a computerized definition of the gear geometries must be obtained and then, in the second step, these gear geometries are discretized into finite elements to obtain the finite element mesh of the

*Corresponding author:

Email address: vroda@uji.es (Victor Roda-Casanova) 
gear drive. The way in which these two steps are carried out has led to different approaches to develop finite element models of gear drives.

In some of these approaches [7, 9, 10], the computerized definition of the gear geometries is obtained by means of a computer aided design (CAD) software. Then, these geometries are imported into a commercial FEA package, where they are discretized into finite elements using proprietary meshing procedures. This is a simple and immediate way to generate finite element meshes of gear transmissions but, as explained by Mao [3], it has some inconveniences that are mainly related to the low accuracy in which the gear geometries are imported into the FEA packages.

In other approaches $[3,5,11]$, the gear geometries are generated directly within the FEA packages, instead of importing them from CAD softwares. For such a purpose, scripts are implemented in the FEA packages, which allow to obtain a mathematical definition of the gear geometries considering the manufacturing process and the gear meshing theory [8]. These approaches allow to overcome the issues related to the low geometrical accuracy of the resulting meshes but, as explained by Hotait [1], they require an important involvement of the user in terms of finite element modeling and geometry details.

For these reasons, a more interesting approach is to develop customized stand-alone computer programs to generate the finite element models of the gear drives $[1,12,13]$. In general, in these computer programs the geometry of the gears is generated directly from the virtual simulation of the manufacturing process. Then, these geometries are discretized into finite elements by means of dedicated meshing procedures. As a result, a finite element mesh of the gear drive is provided, where the position of the nodes of the mesh is defined by the analytical equations of the gear profiles and rim. Besides the gain in the accuracy in which the finite element meshes of the gear drives are generated, this approach allows to ease and automatize the generation of finite element models of the transmission, guarantying the repetitiveness between them.

These computer programs require of the development of dedicated meshing procedures to discretize the gear geometries into finite elements. Among the meshing procedures that have been developed for such a purpose, the one proposed by Argyris et al. [13] is especially relevant, as it has been extensively used by the gearing research community $[4,6,14,15,16]$. This meshing procedure represents a fast and easy way to discretize a gear geometry into finite elements, but it tends to produce distorted elements in some parts of the gear tooth, which may compromise the accuracy of the results of the finite element analysis [17].

Besides that, the meshing procedure proposed by Argyris does not allow to perform local mesh refinement, because it produces symmetric meshes in both driving and coast sides of the gear tooth [18]. However, the ability to perform local mesh refinement is a very convenient feature in a meshing procedure, as it allows to optimize the number of nodes and elements in a finite element mesh and to reduce the computational cost associated to the finite element model. In particular, the benefits of performing local mesh refinement when conducting finite element analyses of gear drives have been illustrated by Barbieri [19] and Liu [20], among others.

Recently, Gonzalez-Perez $[18,21]$ has proposed a new meshing procedure that enhances Argyris' procedure by enabling the performance of local mesh refinement in the vicinity of the contact profile. In this procedure, individual meshes are generated for the refined and the nonrefined areas, and they are connected between them using multi-point constraints. However, using multi-point constraints to connect non-conforming meshes tends to be cumbersome from a model development standpoint [22] and can introduce numerical errors at the mismatching interfaces [23]. Furthermore, the meshing procedure proposed by Gonzalez-Perez does not prevent the generation of distorted elements out of the refined area. 
In spite of the advances that have been done in the field of the finite element analysis of gear drives, the existing meshing procedures to discretize gear geometries into finite elements still have some lacks that need to be overcome. Thus, the objective of this work is to develop a new meshing procedure to perform the automated generation of quadrilateral meshes over gear tooth sections that, being fast and easy to implement, allows to perform local mesh refinement and minimizes the appearance of distorted elements in the mesh.

\section{Definition of the meshing problem and state of the art}

In general, a three-dimensional hexaedral mesh of a gear can be constructed as a rotational pattern of the mesh of a single gear tooth. At the same time, an hexaedral mesh of a gear tooth can be generated by sweeping a two-dimensional quadrilateral mesh of its transverse section along the face width of the gear. For this reason, it can be said that the problem of generating a three-dimensional hexaedral mesh of a gear can be reduced to the problem of generating a two-dimensional quadrilateral mesh over the transverse section of one of its teeth.

For the definition of a tooth section, and without loss of generality, let us consider a transverse section of a typical spur gear as the one shown in Fig. 1a. From this gear section, the tooth section to be meshed is selected (as shaded in gray in Fig. 1a). This tooth section is referred to a local Cartesian coordinate system that, having the origin of coordinates $O_{L}$ in the center of the gear section, has the local $Z_{L}$ axis normal to the section plane. The $Y_{L}$ axis of the local coordinate system is oriented in such a way that the considered tooth section is vertical and centered along this axis.

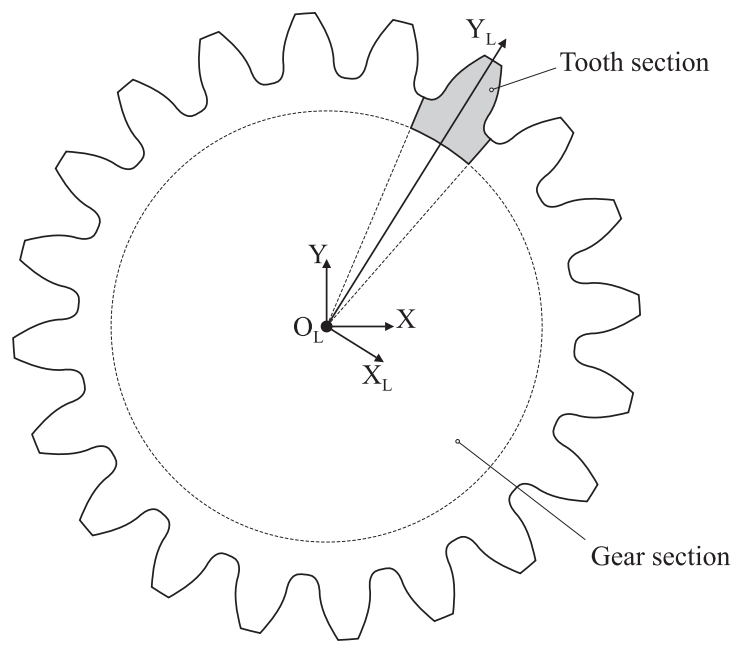

(a) Gear section

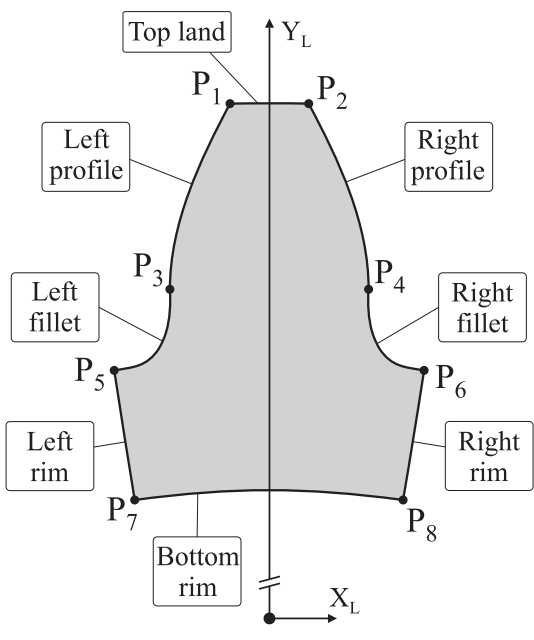

(b) Tooth section

Figure 1: Geometry of a typical gear tooth section

The considered tooth section is shown in greater detail in Fig 1b. It is defined by eight boundary curves that can be analytically determined using the parametric equations of the tooth profile and the portions of the corresponding rim. These curves correspond to the left fillet, left 
profile, top land, right profile, right fillet, right rim, bottom rim and left rim. The positions where two adjacent boundary curves are connected are marked by points $P_{i}\{i=1,2, \ldots, 8\}$.

Generating a quadrilateral mesh over this tooth section is a problem that is not easy to solve, because a tooth section is a non-convex domain with complex boundaries. In these cases there are many researchers $[24,25,26]$ that recommend using multiblock meshing procedures, which can be summarized in the four steps shown in Fig. 2. These four steps have a tight relation between them, which cause the decisions made in each step to have great influence over the rest. For instance, the local meshing scheme that is applied over each patch (step A3) determines not only the geometry allowable for the patch (step A1) but also the type of discretization that is applied to its boundary curves (step A2).

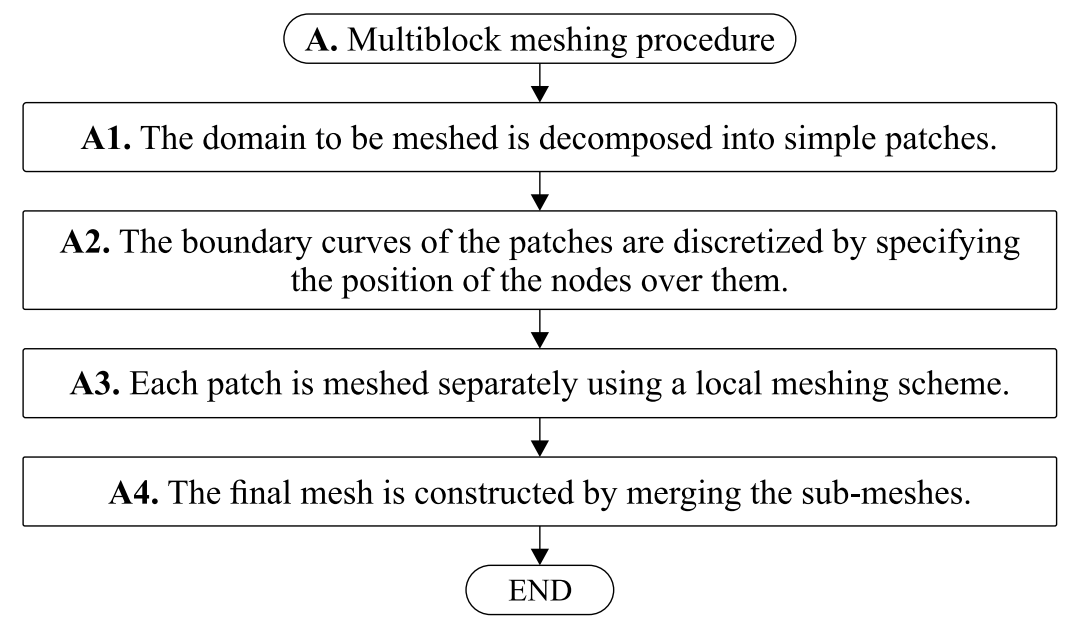

Figure 2: Flowchart of a multiblock meshing procedure

Multiblock meshing procedures have already been used to generate quadrilateral meshes over gear tooth sections, one of the most relevant being the one proposed by Argyris [13]. In this procedure the tooth section is decomposed into six quadrilateral patches, as shown in Fig. 3a. The simplicity of the geometry of the resulting patches allows to use a simple local meshing scheme, as the drag method described by Park [27], to generate a structured quadrilateral mesh directly over each one of them (Fig. 3b). Finally, the meshes of the individual patches are merged together (Fig. 3c), obtaining a quadrilateral mesh for the tooth section.

The method proposed by Argyris is a simple and fast method to mesh a gear tooth section using quadrilateral elements, especially because of the simplicity of the local meshing scheme that is applied to the patches in which the tooth section is decomposed. However, using this local meshing scheme requires opposite boundary curves of the patches having the same number of divisions and some limitations in the meshing process arise as a consequence of this requirement.

On the one hand, the combined effect of the mesh propagation between adjacent patches and the requirement of opposite boundary curves having the same number of divisions implies that local mesh refinement cannot be performed. On the other hand, Argyris' meshing procedure tends to produce distorted elements in parts of the tooth that are usually exposed to elevated gradients of the primary field variable.

In the following sections, a local meshing scheme capable of meshing patches with an arbitrary number of divisions in their boundary curves is described (section 3), which is integrated 


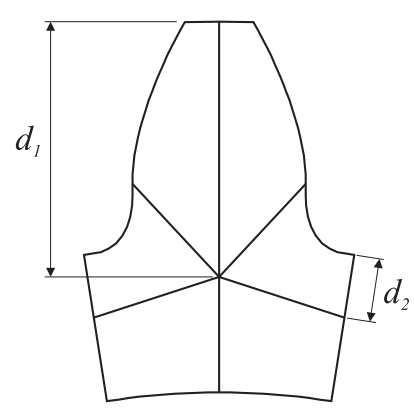

(a)

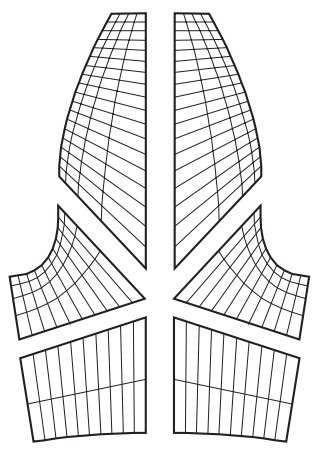

(b)

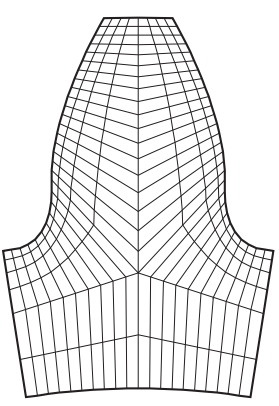

(c)

Figure 3: Argyris' meshing procedure

in the meshing procedure that is proposed in this work (section 4).

\section{Structured mesh generation over a quadrilateral planar patch}

In this section a meshing scheme to generate a structured mesh over a quadrilateral planar patch is described. This scheme is based on the hierarchical template-based recursive decomposition of the domain proposed by Miranda [28], and its main advantage is that it allows to mesh quadrilateral patches having a different number of divisions in opposite boundary curves.

Let us consider a generic planar patch as the one shown in Fig. $4 \mathrm{~b}$, which is defined by four parametric boundary curves that are denoted by $\mathbf{c}_{i}\{i=1,2, \ldots, 4\}$. Over each boundary curve there are distributed $n_{i}+1$ nodes, which represent the position that the nodes of the resulting mesh will occupy over them. These nodes divide each boundary curve in $n_{i}\{i=1,2, \ldots, 4\}$ segments (or divisions).

In general [24], the process of generating a structured quadrilateral mesh over this generic patch can be summarized in the three steps shown in Fig. 4a. In the first step (step B1) the patch is represented in the logical space using a reference square (Fig. 4c), in such a way that each edge of the reference square (which are denoted by $\mathbf{e}_{i}\{i=1,2, \ldots, 4\}$ ) corresponds to a boundary curve of the patch. The nodes over the boundary curves are also represented over the edges of this reference square.

In the second step (step B2), a structured quadrilateral mesh is generated over the reference square (Fig. 4d). For such a purpose, the approach proposed by Miranda [28] is selected, which consist in a hierarchical template-based recursive decomposition of the reference square. Three different templates are considered for this decomposition (denoted by T0, T1 and T2 in Fig. 5), and their selection and orientation depends on the number of divisions in each edge of the square:

- Template T0 is applied when opposite edges have the same number of divisions (Fig. 5a). This template generates one subregion $\left(\mathbf{s}_{A}\right)$ that can be easily meshed using a bilinear interpolation.

- Template T1 is applied when the number of divisions of a pair of opposite edges is equal but the number of divisions of the other pair of opposite edges is different (Fig. 5b). This template generates four subregions $\left(\mathbf{s}_{A}, \mathbf{s}_{B}, \mathbf{s}_{C}, \mathbf{s}_{D}\right)$, where template $\mathrm{T} 0$ is applied. 
B. Local meshing scheme

B1. The patch is represented in the logical space using a reference square.

B2. A structured quadrilateral mesh is generated over the unit-length square in the logical space.

B3. The mesh is transferred from logical space to the quadrilateral patch using mapping functions.

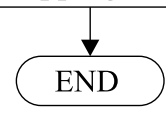

(a) Flowchart

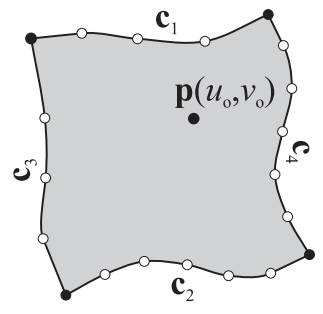

(b) Input information

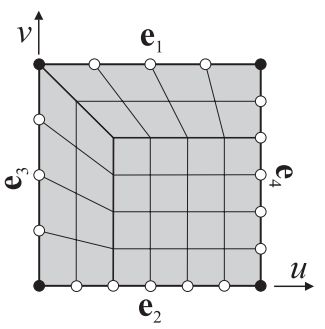

(d) Step B2

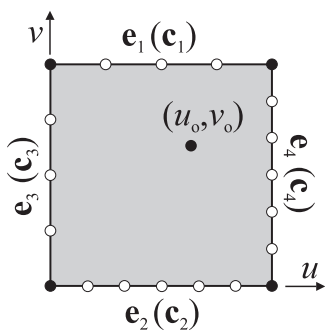

(c) Step B1

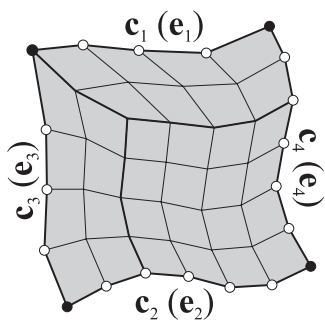

(e) Step B3

Figure 4: Meshing quadrilateral patches using hierarchical templates

- Finally, template T2 is used when the number of divisions in the opposite edges of the square is not equal (Fig. 5c). Template T2 generates three subregions $\left(\mathbf{s}_{A}, \mathbf{s}_{B}, \mathbf{s}_{C}\right)$. Template T0 is applied to subregions $\mathbf{s}_{A}$ and $\mathbf{s}_{B}$. Subregion $\mathbf{s}_{C}$ can be either a T0 or a T1, depending on the number of divisions of each edge of the subregion.

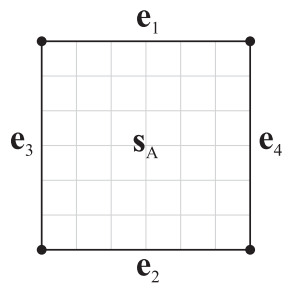

$n_{1}=n_{2}, n_{3}=n_{4}$

(a) Template T0

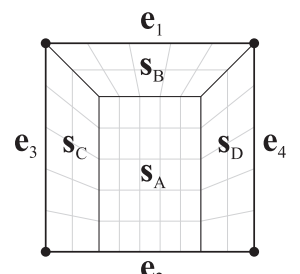

$n_{1}<n_{2}, n_{3}=n_{4}$

(b) Template $\mathrm{T} 1$

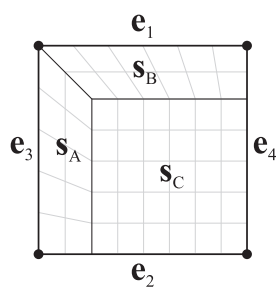

$<n_{2}, n_{3}<n_{4}$

(c) Template T2

Figure 5: Templates used to decompose the reference square and their nomenclature

After applying the template recursive decomposition, template T0 can be applied to all the resulting subregions, so they can be meshed individually using bilinear interpolation. To obtain the final mesh, the meshes of all subregions are merged together.

Finally, in the third step of the local meshing scheme (step B3) the mesh is transferred from the reference square in the logical space to the quadrilateral patch in the physical space using transfinite interpolation [29] (Fig. 4e).

The main advantages of this meshing method are that it is a simple method to generate quadrilateral meshes over patches, it does not require opposite edges of the patch to have the same 
number of divisions, and it generates topologically equivalent meshes for similar patches with similar discretizations of the boundary curves. However, when using this method it is important to bear in mind that the total number of divisions of the boundary curves of the patch must be an even number. In addition, the transfinite interpolation has some limitations $[24,26,30]$ that need to be taken into account:

- The patches must be defined by four parametric boundary curves, being topologically equivalent to a square.

- Discontinuities in the slope of the boundary curves should be avoided, since they may have negative influence on the smoothness of the resulting mesh.

- Non-convex geometries should be avoided as well, since they can produce self-folded meshes.

\section{A new multiblock procedure to mesh gear tooth sections using quadrilateral elements}

In this section a new multiblock procedure to mesh gear tooth sections using quadrilateral elements is proposed. The main goal of this new procedure is to obtain a fast and easy-toimplement meshing method that, keeping the advantages of the existing methods, allows for the performance of local mesh refinement and prevents the appearance of heavily distorted elements in the mesh.

For the development of the proposed meshing procedure it is assumed that the following information is provided in advance:

- The analytical definition of the eight boundary curves (Fig. 1b) that define the tooth section, as well as the points $P_{i}$ that define the intersections between them.

- A discretization of each boundary curve in the form of $n_{i}+1$ nodes, which represent the position that the nodes of the resulting mesh will occupy over them, and constitute the boundary conditions of the meshing problem.

Assuming that this information is given, the proposed meshing procedure follows the four steps presented in Fig. 2. In the first step (step A1) the tooth section is decomposed into simpler patches, following the indications given in section 4.1. Then, the boundary curves of the resulting patches are discretized (step A2) considering the provided meshing boundary conditions and the limitations of the local meshing scheme that is going to be applied over them. Finally, each patch is meshed separately using the local meshing scheme described in section 3 (step A3). The way in which steps $\mathbf{A} \mathbf{2}$ and $\mathbf{A} \mathbf{3}$ are conducted is further explained in sections 4.2 and 4.3, respectively.

After steps A1-A3 are completed, a quadrilateral mesh is obtained for each one of the patches in which the tooth section is decomposed. In the last step (step A4), the resulting meshes are merged together and a quadrilateral mesh for the tooth section is obtained.

\subsection{Step A1: Decomposition of the tooth section in simple patches}

One of the most important things to bear in mind when proposing a decomposition for a gear tooth section is that the geometry of the resulting patches must fulfill the requirements of the local meshing scheme that is going to be applied over them. In the proposed procedure, the 
local meshing scheme described in section 3 will be used to mesh these patches, and thereby the geometry of the patches will be conditioned by its limitations.

But, apart from the limitations imposed by the local meshing scheme, there are other considerations that may have certain influence in the way in which the gear tooth section is decomposed. For example, it is interesting to select a decomposition that minimizes the difference in length of the opposite boundary curves of the patches, as this can help to improve the quality of the resulting meshes. Besides that, it is also interesting to minimize the number of patches in the decomposition, since this simplifies the global meshing process. But, on the other hand, having a larger number of patches allows these patches to have simpler boundaries, which is good from the point of view of the local meshing scheme.

Bearing in mind these limitations and considerations, several ways of decomposing the tooth section have been investigated in depth. Among them, the one that has proven better results is the tooth decomposition shown in Fig. 6. This tooth decomposition is based on the determination of seven auxiliary points, which are denoted by $Q_{i}\{i=9,10, \ldots, 15\}$ (Fig. 7a). Once these auxiliary points are determined, connections between them and the existing points are established using analytical lines, which divide the tooth section into six patches denoted as patch A, B, C, D, E and $\mathrm{F}$ (Fig. 7b). The boundary curves of the resulting patches are denoted by $\mathbf{c}_{i}\{i=1,2, \ldots, 20\}$, as indicated in Fig. 6c.

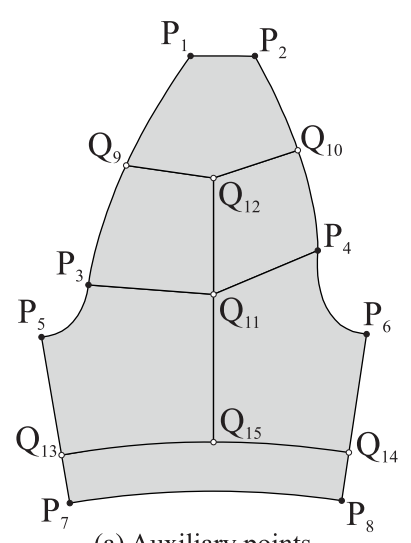

(a) Auxiliary points

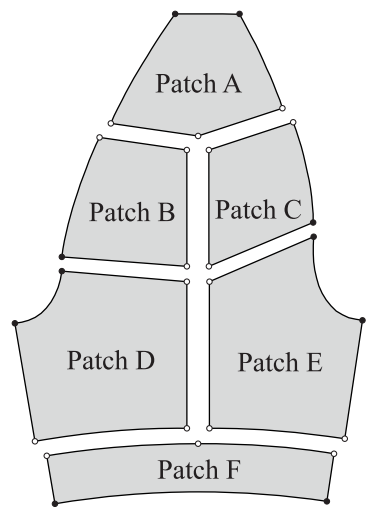

(b) Decomposition in patches

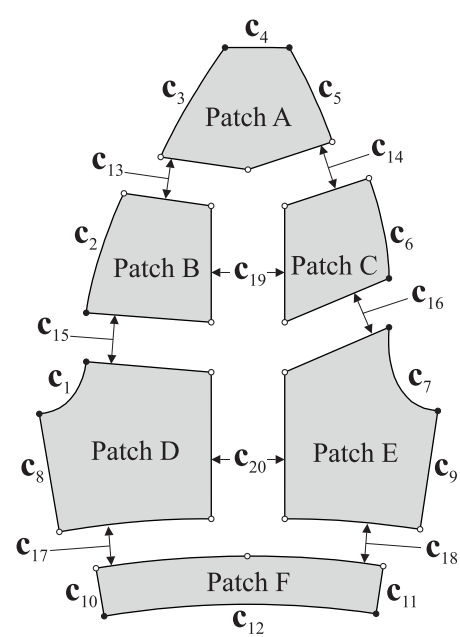

(c) Auxiliary edges

Figure 6: Decomposition of a generic asymmetric tooth section into simpler patches

For the determination of the position of the auxiliary points, the following steps are followed:

i. Points $Q_{9}$ and $Q_{10}$ are located over the left and right profiles (Fig. 1b), respectively, in such a way that they divide these curves in two parts of equal length (Fig. 7a). Then, unit normal vectors at points $P_{3}, P_{4}, Q_{9}$ and $Q_{10}$ are determined (denoted by $\mathbf{u}_{3}, \mathbf{u}_{4}, \mathbf{u}_{9}$ and $\mathbf{u}_{10}$, respectively, in Fig. 7a).

ii. Vector $\mathbf{u}_{L}$ is computed as the bisector of vectors $\mathbf{u}_{3}$ and $\mathbf{u}_{9}$. Similarly, vector $\mathbf{u}_{R}$ is computed as the bisector of vectors $\mathbf{u}_{4}$ and $\mathbf{u}_{10}$.

iii. As shown in Fig. 7b, point $P_{3}$ is projected onto the $Y_{L}$ axis following the direction given by $\mathbf{u}_{L}$ to find point $P_{3}^{\prime}$, and point $P_{4}$ is projected onto the $Y_{L}$ axis following the direction 
given by $\mathbf{u}_{R}$ to find point $P_{4}^{\prime}$. Then, point $Q_{11}$ is located at the midpoint of the segment that connects points $P_{3}^{\prime}$ and $P_{4}^{\prime}$.

iv. Similarly (Fig. 7c), point $Q_{9}$ is projected onto the $Y_{L}$ axis following the direction given by $\mathbf{u}_{L}$ to find point $Q_{9}^{\prime}$, and point $Q_{10}$ is projected onto the $Y_{L}$ axis following the direction given by $\mathbf{u}_{R}$ to find point $Q_{10}^{\prime}$. Then, point $Q_{12}$ is located at the midpoint of the segment that connects points $Q_{9}^{\prime}$ and $Q_{10}^{\prime}$.

v. Distance $d_{A}$ is defined as the average length of segments $\overline{P_{3} Q_{11}}$ and $\overline{P_{4} Q_{11}}$.

vi. Point $Q_{13}$ is located over the left rim at a distance $d_{A}$ of $P_{5}$ and point $Q_{14}$ is located over the right rim at the same distance $d_{A}$ of $P_{6}$, as illustrated in Fig. $7 \mathrm{~d}$.

vii. Finally, point $Q_{15}$ is obtained as the intersection of $Y_{L}$ axis and a circumference centered in $O_{L}$ passing through points $Q_{13}$ and $Q_{14}$ (Fig. 7d).

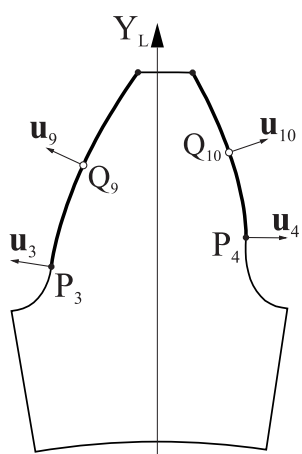

(a)

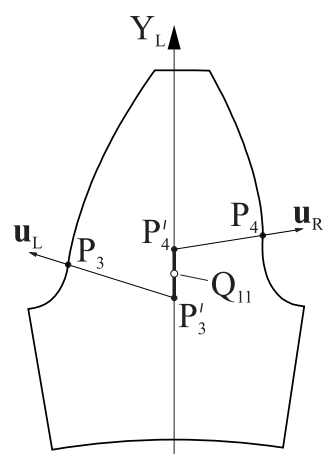

(b)

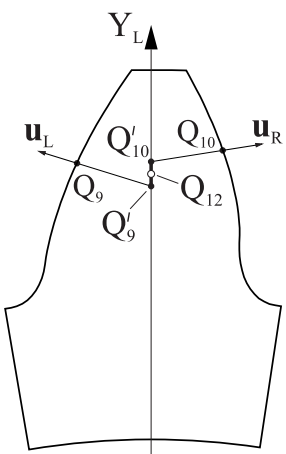

(c)

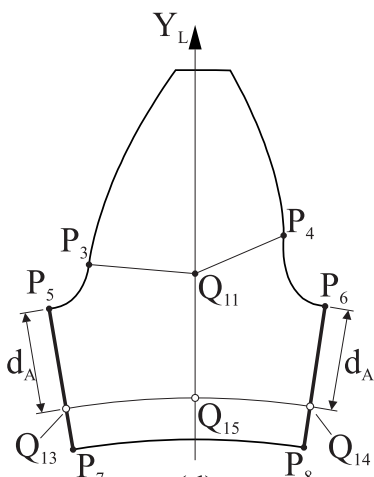

(d)

Figure 7: Determination of the position of the auxiliary points in a generic asymmetric tooth section

Note that there may be some cases in which patch $\mathrm{F}$ will not exist. If distance $d_{A}$ is greater than the length of segments $\overline{P_{5} P_{7}}$ and $\overline{P_{6} P_{8}}$, then patch $\mathrm{F}$ is not considered in the meshing process, and the whole lower part of the tooth corresponds to patches $\mathrm{D}$ and $\mathrm{E}$.

Finally, it is important to mention that the resulting decomposition does not completely match the requirements of the local meshing scheme. On the one hand, patches $\mathrm{A}, \mathrm{D}, \mathrm{E}$ and $\mathrm{F}$ are bounded by more than four boundary curves, and on the other hand, patches D and E are nonconvex regions. These issues must be taken into account in the following steps of the meshing procedure to avoid meshing problems.

\subsection{Step A2: Discretization of the boundary curves of the patches}

As it has been mentioned before, the proposed meshing procedure is developed assuming that the discretization of the boundary curves of the patches is provided in advance, in terms of $n_{i}+1$ nodes that are distributed along each one of these curves, dividing them in $n_{i}$ segments.

However, before continuing with the meshing process, it is necessary to check that the provided discretization is suitable for the local meshing scheme that is going to be applied to the patches. In the proposed procedure, the meshing scheme described in section 3 is used to mesh the patches, and its only requirement is that the total number of divisions in the boundary curves of the patch must be an even number. 
Table 1: General meshing requirements ( $n_{i}$ indicates number of divisions in boundary curve $\mathbf{c}_{i}$ )

\begin{tabular}{ccc}
\hline Requirement & Target patch & Mathematical condition \\
\hline R1 & A & $\left(n_{3}+n_{4}+n_{5}+n_{13}+n_{14}\right)$ must be even \\
R2 & B & $\left(n_{2}+n_{13}+n_{15}+n_{19}\right)$ must be even \\
R3 & C & $\left(n_{6}+n_{14}+n_{16}+n_{19}\right)$ must be even \\
R4 & D & $\left(n_{1}+n_{8}+n_{15}+n_{17}+n_{20}\right)$ must be even \\
R5 & E & $\left(n_{7}+n_{9}+n_{16}+n_{18}+n_{20}\right)$ must be even \\
R6 & F & $\left(n_{10}+n_{11}+n_{12}+n_{17}+n_{18}\right)$ must be even \\
\hline
\end{tabular}

Considering all the patches in which the tooth section is decomposed, this requirement can be turned into the six mathematical conditions shown in Tab. 1. Each one of these conditions is related to one of the patches in which the tooth section is decomposed, and all of them need to be simultaneously fulfilled in order to continue with the meshing process.

\subsection{Step A3: Generation of a quadrilateral mesh over each one of the patches}

The first step when applying the meshing scheme described in section 3 to mesh the patches in which the tooth section is decomposed is to establish a correlation between the boundary curves of each patch in the physical space and the edges of the reference square in the logical space (step B1 in Fig. 4a).

In those patches that are bounded by four boundary curves, such as patches $\mathrm{B}$ and $\mathrm{C}$, a straightforward one-to-one correlation can be established between their boundary curves and the edges of the reference square. In the rest of the patches, which are bounded by five curves, it is necessary to join together two adjacent boundary curves of the patch, and then relate the resulting curve to a single edge of the reference square.

Depending on which adjacent curves are joined together, five different curve combinations can be produced for each patch, and each one of them leads to a different solution for the meshing problem. The selection of the optimum curve combination for each patch depends on which one of them better suits the limitations of the local meshing scheme. In patches A and F, it is clear that the curve combinations shown in Fig. 8a and Fig. 8d are the ones that better suit these limitations, because any other curve combination would present severe discontinuities in the slope of the resulting boundary curves.

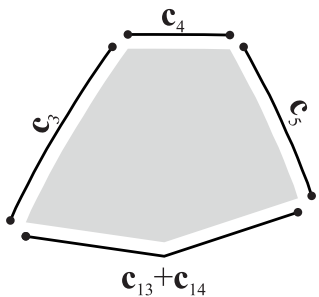

(a) Patch A

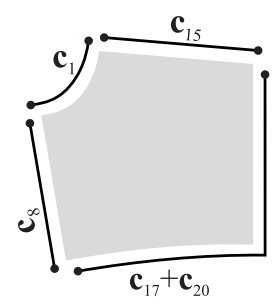

(b) Patch D

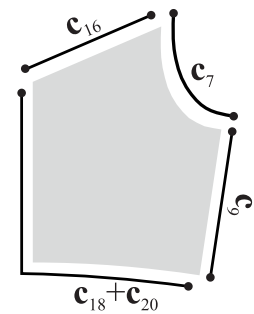

(c) Patch E

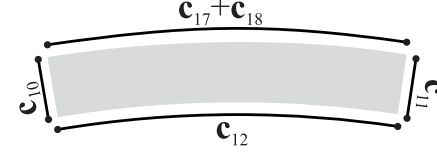

(d) Patch F

Figure 8: Combination of boundary curves for patches A, D, E and F. 
In contrast, in patches $\mathrm{D}$ and $\mathrm{E}$ this election is not as simple, because all the possible curve combinations present similar discontinuities in the slope of the boundary curves and may lead to non-convex geometries. In these cases it is necessary to evaluate all the possible curve combinations and select the one that maximizes the quality of the mapping of the mesh from the logical space to the physical space. In this work, the quality of the mapping has been evaluated through the scaled jacobian [31] of the transfinite interpolation, an it has revealed that the optimal curve combinations for these patches are the ones shown in Fig. $8 \mathrm{~b}$ and Fig. 8c.

Once the correlation between the boundary curves of the patches in the physical space and the edges of the reference square in the logical space is established, the steps $\mathbf{B} 2$ and $\mathbf{B 3}$ of the local meshing scheme can be followed to produce a quadrilateral mesh over each patch.

\section{Improving the quality of the mesh}

When performing a finite element analysis, the geometry of the elements in which the domain of the problem is discretized is a factor that has an important influence over the accuracy of the obtained results. It is well known that the presence of distorted elements reduces the accuracy of the results of the analysis, especially when these distorted elements are located in areas where elevated gradients of the primary field variable are produced. In consequence, distorted elements should be avoided in the whole mesh in general, and in particular in those regions of the gear tooth where elevated gradients of the primary field variable are expected.

Unfortunately, quadrilateral mesh generation algorithms (as the one proposed in this work) tend to produce meshes that contain distorted elements. This is the reason why they are usually completed with mesh quality enhancement techniques. In this work, two different techniques have been considered:

- An a priori mesh quality enhancement technique, which is explained in section 5.1.

- An a posteriori mesh quality enhancement technique, which is explained in section 5.2.

\subsection{A priori enhancement of the quality of the mesh}

In the proposed meshing procedure, the mesh of the gear tooth is generated by merging the individual meshes of the patches in which the tooth section is decomposed. In consequence, separate actions can be taken to avoid the appearance of distorted elements in each one of the patches.

The generation of distorted elements when meshing a given patch depends on the meshing template that is applied by the local meshing scheme. In general, when a meshing template produces distorted elements in the reference square in the logical space (see templates T1 and T2 in Fig. 5), these distorted elements tend to be transferred to the patch in the physical space. In addition, the orientation of the meshing template determines the region of the patch where these distorted elements appear. This fact is illustrated in Fig. 9, where all the possible orientations of meshing templates $\mathrm{T} 0, \mathrm{~T} 1$ and $\mathrm{T} 2$ have been used to mesh patch $\mathrm{D}$ of the gear tooth section.

As explained in section 3, the selection and the orientation of the meshing templates depends on the number of divisions in the boundary curves of the patch. Hence, the appearance of distorted elements can be controlled by selecting an appropriate number of divisions in the boundary curves of the patch. This can be done by adding meshing conditions when discretizing the boundary curves of the patches, which are added to the meshing requirements shown in Tab. 1. 


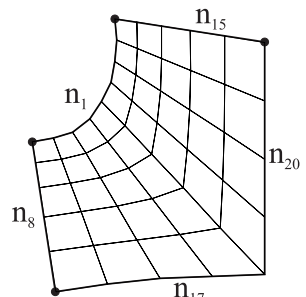

(a) Template T0

$\mathrm{n}_{1}=\mathrm{n}_{17}+\mathrm{n}_{20}, \mathrm{n}_{8}=\mathrm{n}_{15}$

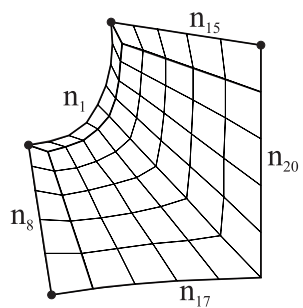

(d) Template $\mathrm{T} 1$

$\mathrm{n}_{1}<\mathrm{n}_{17}+\mathrm{n}_{20}, \mathrm{n}_{8}=\mathrm{n}_{15}$

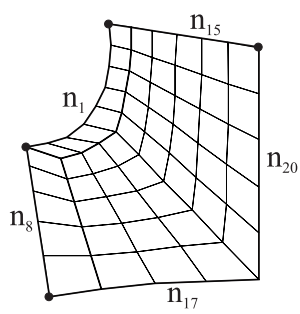

(g) Template T2

$\mathrm{n}_{1}<\mathrm{n}_{17}+\mathrm{n}_{20}, \mathrm{n}_{8}<\mathrm{n}_{15}$

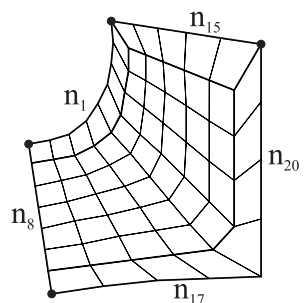

(b) Template $\mathrm{T} 1$

$\mathrm{n}_{1}=\mathrm{n}_{17}+\mathrm{n}_{20}, \mathrm{n}_{8}>\mathrm{n}_{15}$

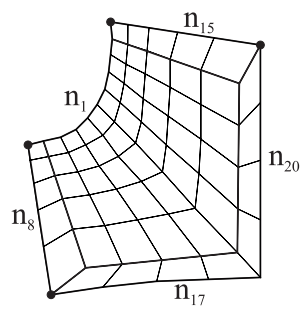

(e) Template $\mathrm{T} 1$

$\mathrm{n}_{1}>\mathrm{n}_{17}+\mathrm{n}_{20}, \mathrm{n}_{8}=\mathrm{n}_{15}$

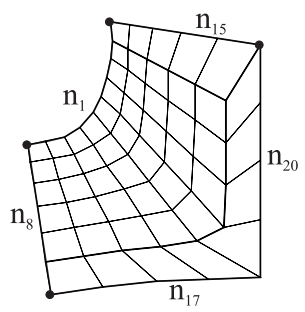

(h) Template T2

$\mathrm{n}_{1}>\mathrm{n}_{17}+\mathrm{n}_{20}, \mathrm{n}_{8}>\mathrm{n}_{15}$

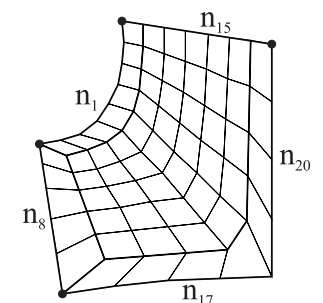

(c) Template $\mathrm{T} 1$

$\mathrm{n}_{1}=\mathrm{n}_{17}+\mathrm{n}_{20}, \mathrm{n}_{8}<\mathrm{n}_{15}$

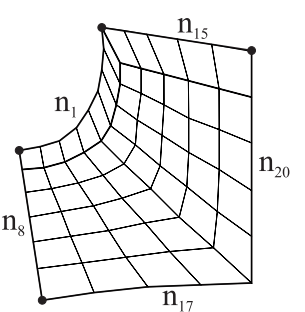

(f) Template T2

$\mathrm{n}_{1}<\mathrm{n}_{17}+\mathrm{n}_{20}, \mathrm{n}_{8}>\mathrm{n}_{15}$

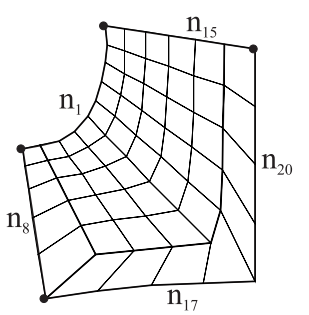

(i) Template $\mathrm{T} 2$

$\mathrm{n}_{1}>\mathrm{n}_{17}+\mathrm{n}_{20}, \mathrm{n}_{8}<\mathrm{n}_{15}$

Figure 9: Meshing templates applied to patch D 
Table 2: Meshing conditions to improve the quality of the mesh $\left(n_{i}\right.$ indicates number of divisions in boundary curve $\left.\mathbf{c}_{i}\right)$

\begin{tabular}{ccc}
\hline Condition & Target patch & Mathematical condition \\
\hline C1 & D & $\begin{array}{c}n_{1} \geq n_{17}+n_{20} \\
\text { if } n_{1}=n_{17}+n_{20} \rightarrow n_{8}=n_{15}\end{array}$ \\
\hline C2 & E & $\begin{array}{c}n_{7} \geq n_{18}+n_{20} \\
\text { if } n_{7}=n_{18}+n_{20} \rightarrow n_{9}=n_{16}\end{array}$ \\
\hline C3 & F & $\begin{array}{c}n_{10}=n_{11} \\
n_{17}+n_{18}=n_{12}\end{array}$ \\
\hline
\end{tabular}

To provide an example, let us focus firstly in patch D, which is used to mesh the left part of the rim of the gear tooth. In this patch it is interesting to avoid the appearance of distorted elements in the vicinity of the fillet, as elevated gradients of the primary field variable are usually produced in this region. As it can be observed in Fig. 9, this can be achieved by two different ways:

- by setting $n_{1}>n_{17}+n_{20}$, regardless of the value of $n_{8}$ and $n_{15}$ (Fig. 9e, h and i),

- by setting $n_{1}=n_{17}+n_{20}$, as long as $n_{8}=n_{15}$ (Fig. 9a).

Both mathematical conditions are included in the meshing condition $\mathrm{C} 1$ shown in Tab. 2. A similar reasoning can be made for patch E, leading to the meshing condition C2 shown in Tab. 2 .

Patches A, B and C are used to mesh the superior part of the gear tooth, including the contact profiles. As a consequence of the gear tooth contact, the contact profiles are regions exposed to elevated gradients of the primary field variable, and in consequence it is also interesting to avoid the appearance of distorted elements when meshing these patches. However, unlike in patches D and $\mathrm{E}$, it is not easy to provide general meshing conditions for these patches without introducing excessive limitations in the meshing process. For this reason, it is left to the user to provide meshing boundary conditions that minimize the appearance of distorted elements.

However, two meshing recommendations can be given in order to minimize the generation of distorted elements in these patches. On the one hand, the user should reduce, as much as possible, the difference between the number of divisions of opposite boundary curves of the patches. On the other hand, the user should specify smooth transitions between the number of divisions in the opposite boundary curves of adjacent patches, in order to avoid unbalanced meshes. For example, when specifying the number of divisions in curves $\mathbf{c}_{13}$ and $\mathbf{c}_{14}$, the user should consider the number of divisions specified for boundary curves $\mathbf{c}_{4}, \mathbf{c}_{15}$ and $\mathbf{c}_{16}$ :

$$
n_{13}+n_{14} \simeq \frac{n_{4}+\left(n_{15}+n_{16}\right)}{2}
$$

Finally, patch D is used to mesh the lower rim of the tooth. In the great majority of the cases, this part of the tooth is not subjected to elevated gradients of the primary field variable, and in consequence it is not usually affected by special meshing requirements. With the aim of improving the general quality of the mesh of the tooth, it is interesting to ensure that template T0 is used to mesh this patch, which can be done by applying the meshing condition C3 from Tab. 2 . 
These measures to improve the quality of the resulting mesh do not carry an increase of the computational cost of the meshing procedure. Although they limit the flexibility of the user, they are not a requirement of the meshing procedure and can be ignored whenever it is needed.

\subsection{A posteriori enhancement of the quality of the mesh}

The objective of the a posteriori mesh quality enhancement techniques is to reduce the distortion of the elements once the mesh is generated. In general, they can be classified into clean-up and mesh smoothing techniques. While the former improve the quality of the mesh by changing the connectivity of its elements, the latter reduce the distortion of the elements by varying the position of its inner nodes.

Mesh smoothing techniques are preferred in this work, as they are especially indicated to reduce the distortion of the elements of the mesh. There is a wide range of methods to perform mesh smoothing, which go from Laplacian to optimization-based methods. While the former are more suitable in terms of computational cost, the latter lead to better quality meshes. A big number of methods have arisen as a mixture of them, that combine features of both types of technique (a survey on these methods can be found in Ref. [25]).

Among these hybrid techniques, the one proposed by Zhou [32] has been selected for this work because it offers three interesting features: it provides better results than the Laplacian smoothing, its computational cost is lower than the objective-based smoothing and it is easy to implement. This mesh smoothing consist of an iterative algorithm whose main flowchart is shown in Fig. 10a. For each iteration of the algorithm, each interior node $m$ of the mesh is sequentially moved to its corresponding optimum position, and its corresponding nodal displacement $\delta_{m}$ is computed. This process is continued until the maximum displacement of the inner nodes is below a certain tolerance $\delta_{t o l}$ or a maximum number of iterations $i_{\text {max }}$ is reached.
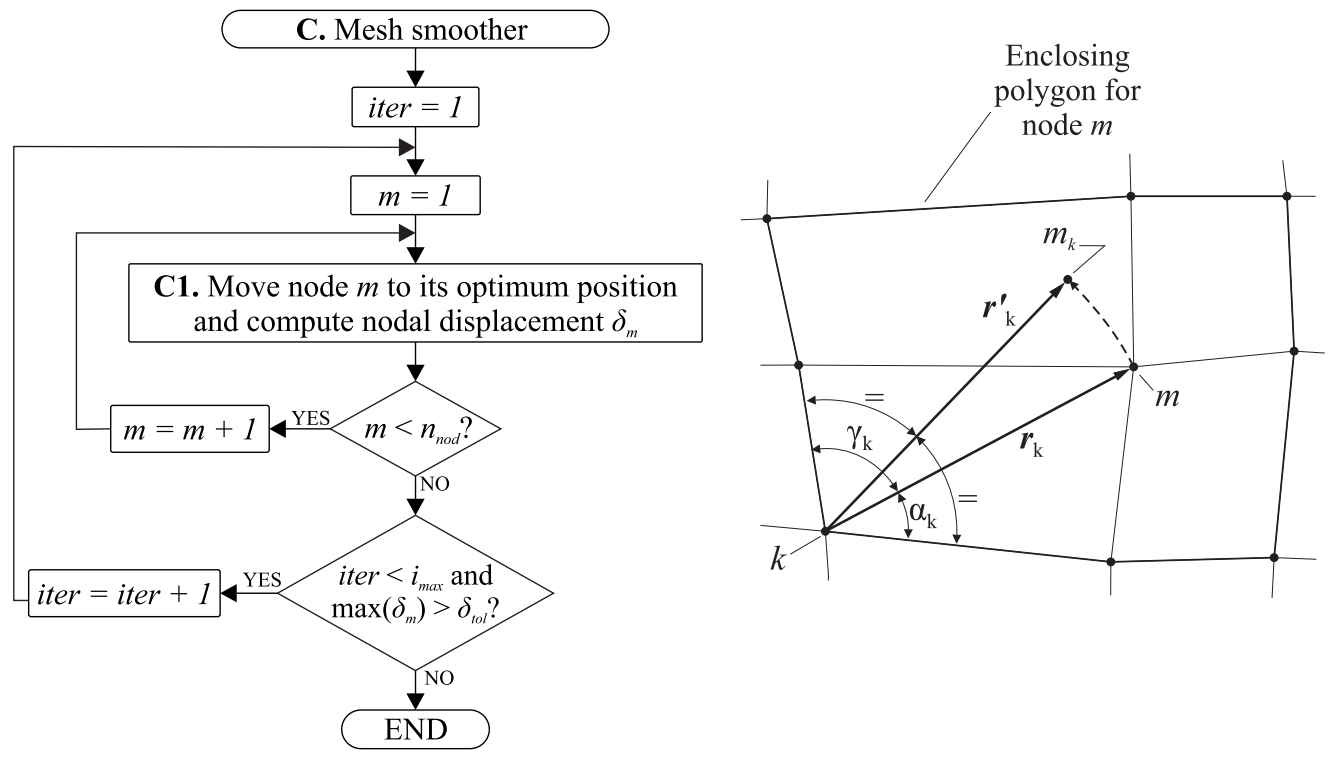

(a) Flowchart of the mesh smoother

(b) Optimization of the position of node $m$

Figure 10: Mesh smoothing 
For the definition of the optimum position of node $m$, consider a sample quadrilateral mesh as the one shown in Fig. 10b. Let $m$ be an interior node of the mesh, and $k$ any of the nodes of the enclosing polygon around $m$. Vector $\mathbf{r}_{k}$ describes the position of node $m$ with respect to node $k$, and $\alpha_{k}$ and $\gamma_{k}$ are the angles between vector $\mathbf{r}_{k}$ and the edges of the enclosing polygon at node $k$. On the other hand, $m_{k}$ represents the improved position of node $m$ with respect to node $k$, which is obtained by rotating the vector $\mathbf{r}_{k}$ around point $k$ to fulfill $\alpha_{k}=\gamma_{k}$. A collection of improved positions $m_{k}$ is obtained for each interior node $m$ of the mesh, one for each node $k$ of its enclosing polygon. The optimum position of node $m$ is defined as the average of this collection of improved positions $m_{k}$.

\section{Numerical examples and discussion of results}

The performance of the proposed procedure is illustrated by meshing several cases of study that are obtained as a combination of a gear geometry and a boundary curve discretization. Two different gear geometries are considered in the study:

- Gear geometry G1 corresponds to a standard spur gear, with $z=20$ teeth, modulus $m=2 \mathrm{~mm}$, pressure angle $\alpha=20^{\circ}$, addendum of standard basic rack tooth $h_{a P}=2 \mathrm{~mm}$, dedendum of standard basic rack tooth $h_{f P}=2.5 \mathrm{~mm}$ and tooth depth $h=4.5 \mathrm{~mm}$.

- Gear geometry G2 corresponds to a spur gear with pointed teeth, having $z=20$ teeth, modulus $m=2 \mathrm{~mm}$, pressure angle $\alpha=25^{\circ}$, profile shift coefficient $x=0.5$, addendum of standard basic rack tooth $h_{a P}=2 \mathrm{~mm}$, dedendum of standard basic rack tooth $h_{f P}=2.5 \mathrm{~mm}$ and tooth depth $h=4.5 \mathrm{~mm}$.

Both gear geometries are meshed considering two different discretizations of the boundary curves, which are denoted by FM (fine mesh) and CM (coarse mesh):

- In the boundary discretization FM a uniform element size equal to $0.15 \mathrm{~mm}$ has been specified for all the external curves of the tooth section (left fillet, left profile, top land, right profile and right fillet). The rest of the curves are discretized following geometric progressions, with a maximum element size equal to $0.30 \mathrm{~mm}$.

- In the boundary discretization CM a uniform element size equal to $0.30 \mathrm{~mm}$ has been specified for all the external curves of the tooth section. The rest of the curves are discretized following geometric progressions, with a maximum element size equal to $0.60 \mathrm{~mm}$.

Table 3 summarizes the cases of study that have been considered in this investigation. Each case of study can be meshed using three different variations of the proposed meshing procedure:

- A standard meshing procedure ST, in which the proposed meshing procedure is applied without any mesh quality enhancement technique.

- A pre-improved meshing procedure PI, in which the proposed meshing procedure is applied considering the a priori mesh quality enhancement technique described in section 5.1 .

- A double-improved meshing procedure DI, in which the pre-improved meshing procedure is completed with the a posteriori mesh quality enhancement technique described in section 5.2. In this procedure the number of iterations of the mesh smoothing algorithm has been limited to 10 and the maximum displacement to $0.02 \mathrm{~mm}$. 
Table 3: Summary of selected cases of study and resulting meshes

\begin{tabular}{|c|c|c|c|c|c|}
\hline $\begin{array}{l}\text { Case of } \\
\text { study }\end{array}$ & $\begin{array}{c}\text { Gear } \\
\text { geometry }\end{array}$ & $\begin{array}{c}\text { Boundary } \\
\text { discretization }\end{array}$ & $\begin{array}{c}\text { Resulting } \\
\text { mesh }\end{array}$ & $\begin{array}{l}\text { Meshing } \\
\text { procedure }\end{array}$ & Figure \\
\hline \multirow{3}{*}{ G1FM } & \multirow{3}{*}{ G1 } & \multirow{3}{*}{ FM } & G1FM-ST & Standard & $11 \mathrm{a}$ \\
\hline & & & G1FM-PI & Pre-improved & $11 \mathrm{~b}$ \\
\hline & & & G1FM-DI & Double-improved & $11 \mathrm{c}$ \\
\hline \multirow{3}{*}{ G1CM } & \multirow{3}{*}{ G1 } & \multirow{3}{*}{$\mathrm{CM}$} & G1CM-ST & Standard & $11 \mathrm{~d}$ \\
\hline & & & G1CM-PI & Pre-improved & $11 \mathrm{e}$ \\
\hline & & & G1CM-DI & Double-improved & $11 \mathrm{f}$ \\
\hline \multirow{3}{*}{ G2FM } & \multirow{3}{*}{ G2 } & \multirow{3}{*}{ FM } & G2FM-ST & Standard & $12 \mathrm{a}$ \\
\hline & & & G2FM-DI & Pre-improved & $12 \mathrm{~b}$ \\
\hline & & & G2FM-PI & Double-improved & $12 \mathrm{c}$ \\
\hline \multirow{3}{*}{$\mathrm{G} 2 \mathrm{CM}$} & \multirow{3}{*}{ G2 } & \multirow{3}{*}{$\mathrm{CM}$} & G2CM-ST & Standard & $12 \mathrm{~d}$ \\
\hline & & & G2CM-PI & Pre-improved & $12 \mathrm{e}$ \\
\hline & & & G2CM-DI & Double-improved & $12 \mathrm{f}$ \\
\hline
\end{tabular}

As a result of meshing the selected cases of study using these variations of the proposed meshing procedure, the 12 meshes reflected in Tab. 3 have been obtained. These meshes are compared between them in terms of:

- The number of nodes and the number of elements of the mesh, as these parameters are relevant to the computational cost associated to the solution of the finite element model.

- The quality of the mesh, represented by the average and the maximum distortion of the elements of the mesh, as there is a relation between the distortion of the elements and the accuracy of the solution of the finite element model.

The distortion of a given element can be defined as the degree of deviation of that element compared to the regular reference element, which, in the case of quadrilateral meshes, is a square. To measure how much an element is distorted, mesh quality metrics are used. Among the several mesh quality metrics available in the literature, Oddy's metric [33] has been selected in this work because it is a robust metric that allows to consider all the types of element distortion (shearing, stretching and combinations of both) in just one value.

Oddy's metric $f_{O}$ is calculated for every element of the mesh following the equation presented in Ref. [33]. When the evaluated element is not distorted (i.e. it is a square) the value of Oddy's metric is zero, and this value increases to infinity as it does the distortion of the element. The maximum distortion of the elements of the mesh is defined by the maximum value of Oddy's metric $f_{O, \max }$ and the average distortion of the elements of the mesh is defined by the average value of Oddy's metric $f_{O, \text { avg }}$.

Figure 11 shows the meshes that are obtained when cases of study G1FM and G1CM are meshed using the standard, the pre-improved and the double-improved meshing procedures. A grayscale code has been used to indicate the amount of distortion of the elements of each mesh, represented by Oddy's metric $\left(f_{O}\right)$, in which the color of the elements becomes darker as their distortion increases. 


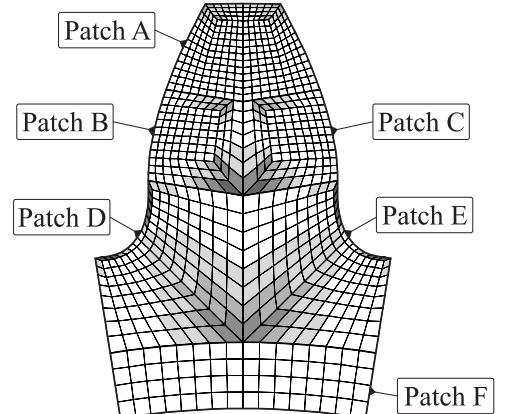

(a) G1FM-ST

838 elem. / 902 nodes

$f_{o, \text { max }}=42.9 / f_{\text {o,avg }}=2.0$

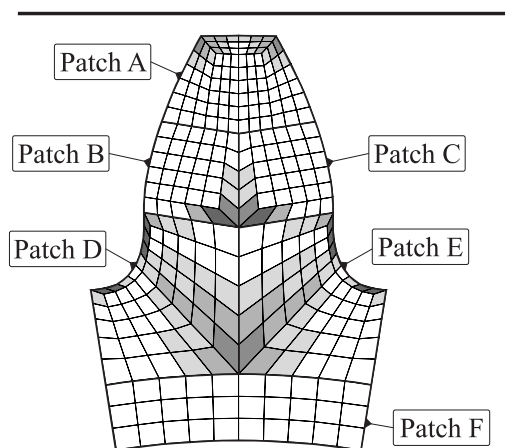

(d) G1CM-ST

306 elem. / 343 nodes

$f_{O, \text { max }}=33.2 / f_{O \text {,avg }}=2.5$

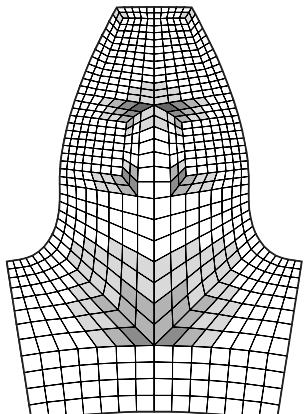

(b) G1FM-PI

678 elem. $/ 741$ nodes

$f_{O, \max }=11.7 / f_{O, \text { avg }}=1.5$

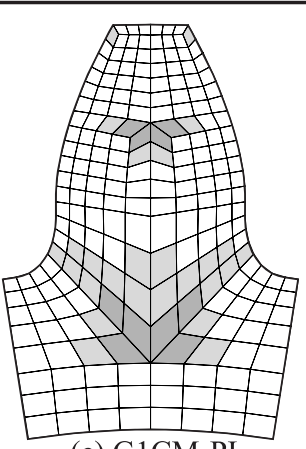

(e) G1CM-PI

216 elem. $/ 252$ nodes

$f_{O \text {, } \max }=7.4 / f_{O, \text { avg }}=1.4$

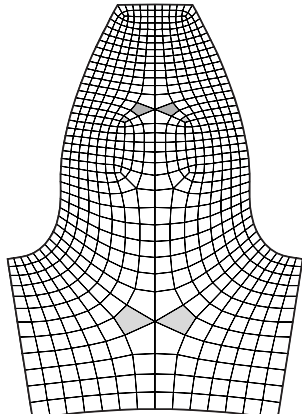

(c) G1FM-DI

678 elem. / 741 nodes $f_{O \text {, } \max }=4.9 / f_{O, \text { avg }}=0.6$
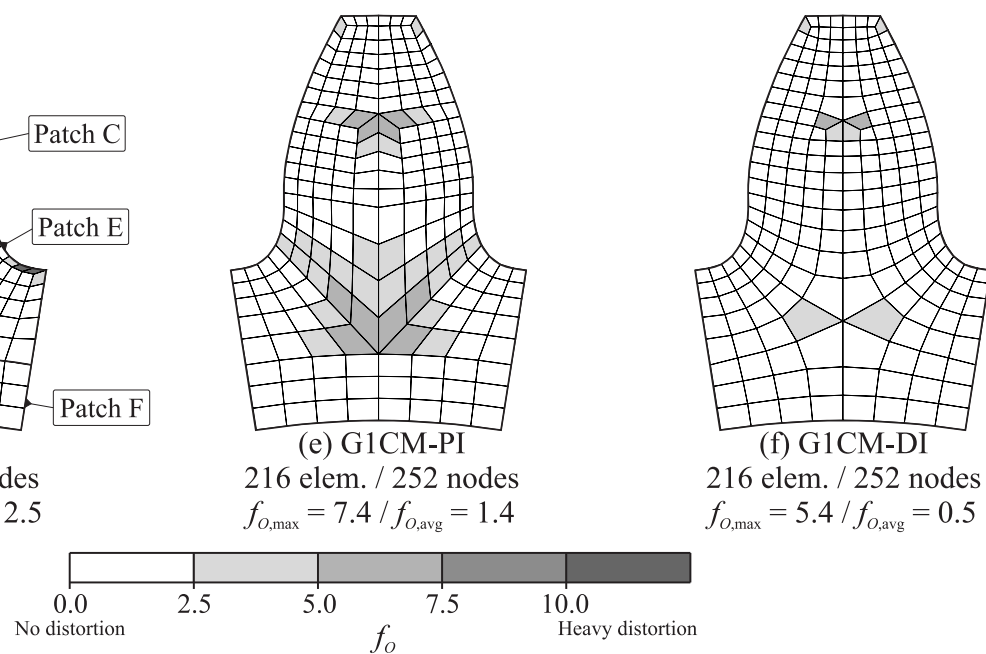

(f) G1CM-DI

216 elem. $/ 252$ nodes $f_{O, \text { max }}=5.4 / f_{O, \text { avg }}=0.5$

Figure 11: Meshes obtained for cases of study G1FM and G1CM 
The meshes that are obtained when the standard meshing procedure is used to mesh cases of study G1FM and G1CM are shown in Fig. 11a and Fig. 11d, respectively. These figures demonstrate that this procedure can be successfully used to discretize gear geometry G1 into quadrilateral elements, either using a fine mesh (G1FM-ST) or a coarse mesh (G1CM-ST). However, it is observed that in both cases it tends to generate distorted elements in the vicinity of the fillets (patches D and E), in the central part (patches B and C) and near the tip of the tooth (patch A).

These distorted elements can be partially avoided by meshing these cases of study using the pre-improved meshing procedure. Figure $11 \mathrm{~b}$ shows the mesh that is obtained when case of study G1FM is meshed using this procedure (G1FM-PI). The visual comparison between this mesh and the one obtained using the standard procedure (G1FM-ST, Fig. 11a) reveals the benefits of using the a priori mesh quality enhancement technique, as the distortion of the elements in the vicinity of the fillets and at the tip of the tooth is reduced. In this particular case of study the consideration of the a priori mesh quality enhancement technique brings a reduction of the $71 \%$ of the maximum distortion $\left(f_{O, \max }\right)$ and a reduction of the $24 \%$ of the average distortion $\left(f_{O, \text { avg }}\right)$ of the mesh, without a noticeable increase of the computational time associated to the procedure. Similar results are observed when case of study G1CM is meshed using the preimproved meshing procedure (G1CM-PI, Fig. 11e).

The application of the a priori mesh quality enhancement technique does not severely affect the original meshing boundary conditions, as only minor changes need to be applied to the discretization of the boundary curves of the patches to fulfill the meshing conditions described in section 5.1. As a result of these changes, a reduction of the number of nodes and elements in the mesh is achieved, which does not affect to the mesh density in the fillets nor the contact profiles.

Finally, the double-improved meshing procedure can be applied to maximize the quality of the meshes. Examples of the meshes that are obtained when cases of study G1FM and G1CM are meshed using this procedure are shown in Fig. 11c (G1FM-DI) and Fig. 11f (G1CM-DI), respectively. In these cases, the application of the a posteriori mesh quality enhancement technique brings reductions over the $55 \%$ of the maximum and the average distortion of the meshes. The number of nodes and elements in the meshes is not affected by mesh smoothing, because this technique varies the position of the nodes but does not change either the connectivity or the number of nodes of the discretization. However, it is important to remark that unlike the a priori mesh quality enhancement technique, the a posteriori mesh quality enhancement technique increases the computational time associated to the meshing procedure.

Considering an average workstation, the computer time associated to the pre-improved meshing procedure has been lower than 1 second in all the studied cases. For a given case of study, the computer time associated to the double-improved meshing procedure is approximately three times the computer time associated to the pre-improved procedure. The difference in computer times resides in the time associated to the a posteriori mesh quality enhancement technique, which is proportional to the number of nodes in the mesh and the number of iterations of the mesh smoother.

A similar discussion can be made for the meshes that are obtained when cases of study G2FM and $\mathrm{G} 2 \mathrm{CM}$ are meshed using the standard, the pre-improved and the double-improved meshing procedures, which are shown in Fig. 12. In general, the quality of these meshes is slightly worse than the quality of the meshes obtained for cases of study G1FM and G1CM (Fig. 11), as gear geometry G2 presents higher difficulties for the meshing procedures. These difficulties reside in the fact that there is a larger difference between the length of the opposite boundary curves of the patches in which the tooth section is decomposed. This fact is illustrated in Tab. 4, where the ratios between the length of the opposite boundary curves of the patches are shown for gear 
Table 4: Ratios between the length of opposite boundary curves of the patches

\begin{tabular}{|c|c|c|c|}
\hline Patch & Curves & Geometry G1 & Geometry G2 \\
\hline \multirow{2}{*}{ A } & length $\left(\mathbf{c}_{13}+\mathbf{c}_{14}\right) /$ length $\left(\mathbf{c}_{4}\right)$ & 2.06 & 3.72 \\
& length $\left(\mathbf{c}_{3}\right) /$ length $\left(\mathbf{c}_{5}\right)$ & 1.00 & 1.00 \\
\hline \multirow{2}{*}{ B, C } & length $\left(\mathbf{c}_{13}\right) /$ length $\left(\mathbf{c}_{15}\right)$ & 1.24 & 1.42 \\
& length $\left(\mathbf{c}_{2}\right) /$ length $\left(\mathbf{c}_{19}\right)$ & 1.00 & 1.04 \\
\hline \multirow{2}{*}{ D, E } & length $\left(\mathbf{c}_{17}+\mathbf{c}_{20}\right) /$ length $\left(\mathbf{c}_{1}\right)$ & 2.62 & 4.77 \\
& length $\left(\mathbf{c}_{8}\right) /$ length $\left(\mathbf{c}_{15}\right)$ & 1.00 & 1.00 \\
\hline \multirow{2}{*}{$\mathrm{F}$} & length $\left(\mathbf{c}_{17}+\mathbf{c}_{18}\right) /$ length $\left(\mathbf{c}_{12}\right)$ & 1.08 & 1.05 \\
& length $\left(\mathbf{c}_{10}\right) /$ length $\left(\mathbf{c}_{11}\right)$ & 1.00 & 1.00 \\
\hline
\end{tabular}

geometries G1 and G2. As it can be observed, these ratios are larger for gear geometry G2 than for gear geometry G1, especially for patches A, D and E.

\subsection{Local mesh refinement}

In this section the ability of the proposed procedure to perform local mesh refinement is checked. For such a purpose, two additional cases of study have been developed, in which gear geometry G1 is meshed under boundary discretizations that lead to local mesh refinement at the fillet (case of study G1FR) and at the center of the contact profile (case of study G1PR). The resulting meshes could be useful to evaluate the bending stress at the tooth root and the contact stress that arise from the bearing contact, respectively.

Figures 13a and 13c show the meshes that are obtained when these cases are meshed using the pre-improved meshing procedure (G1FR-PI and G1PR-PI, respectively). In both cases of study a uniform element size equal to $0.30 \mathrm{~mm}$ is specified for the external curves of the tooth section, except for the mesh refinement area, where a uniform element size equal to $0.15 \mathrm{~mm}$ is defined. In case of study G1FR there is an abrupt change between the element size in the refined area and the element size in the non-refined area, whereas in case of study G1PR there is a smooth transition between the element size in both areas, where the size of the elements increases following a geometric growth.

In meshes G1FR-PI and G1PR-PI, the number of nodes and elements are in the same order of magnitude that in mesh G1CM-PI (Fig. 11e), but the mesh density in those parts of the tooth where the local mesh refinement is performed is within the range of the mesh G1FM-PI (Fig. 11b). This means that the computational time associated to the solution of these finite element models is similar to the computational time associated to the coarse mesh G1CM-PI, but the accuracy of the results of the analysis within the refined areas will be similar to the accuracy of the results obtained with the fine mesh G1FM-PI.

However, the application of local mesh refinement brings a worsening of the quality of the mesh. Compared to meshes G1FM-PI and G1CM-PI, meshes G1FR-PI and G1PR-PI show larger values of average and maximum distortion, and heavily distorted elements appear in those patches that are affected by the local mesh refinement. This happens because these patches need to accommodate larger variations in the number of divisions of their opposite boundary curves.

The distortion of these meshes can be reduced using a posteriori mesh quality enhancement techniques. Thus, Figs. $13 \mathrm{~b}$ and $13 \mathrm{~d}$ show the meshes that are obtained when cases of 


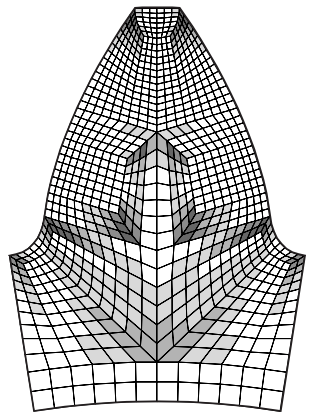

(a) G2FM-ST 892 elem. / 950 nodes $f_{o, \max }=26.3 / f_{o, \text { avg }}=2.4$

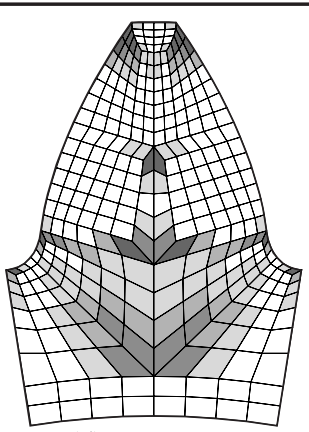

(d) G2CM-ST

318 elem. $/ 353$ nodes

$f_{o, \text { max }}=31.7 / f_{o, \text { avg }}=3.0$

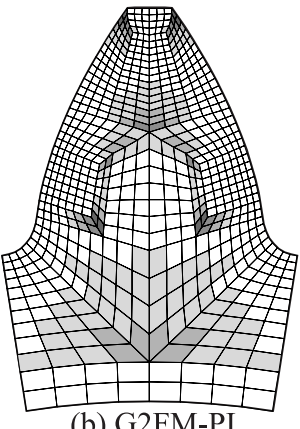

(b) G2FM-PI 662 elem. $/ 718$ nodes $f_{o, \text { max }}=17.4 / f_{o, \text { avg }}=2.0$

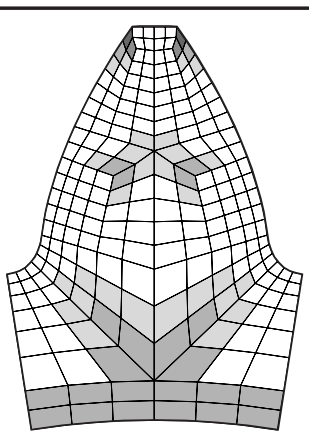

(e) G2CM-PI

224 elem. $/ 258$ nodes

$f_{\text {, } \max }=15.2 / f_{O \text {,avg }}=2.0$

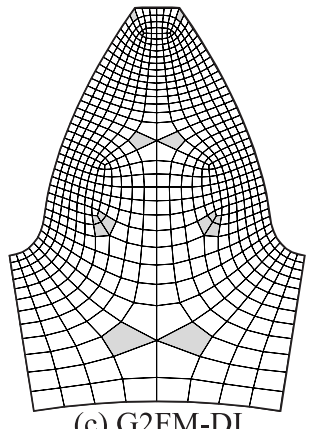

(c) G2FM-DI 662 elem. $/ 718$ nodes $f_{o, \text { max }}=3.9 / f_{O, \text { avg }}=0.5$

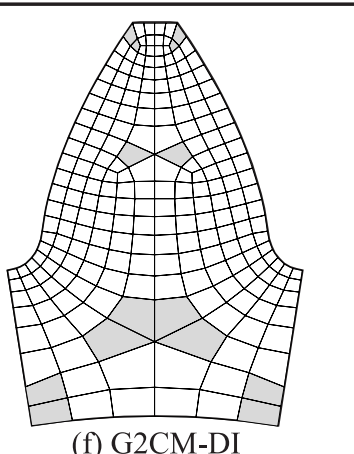

(f) G2CM-D

224 elem. $/ 258$ nodes

$f_{o, \text { max }}=4.6 / f_{O, \text { avg }}=0.6$

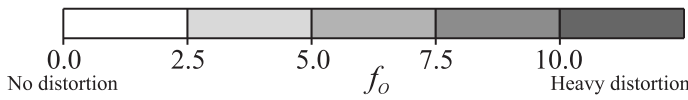

Figure 12: Meshes obtained for cases of study G2FM and G2CM 


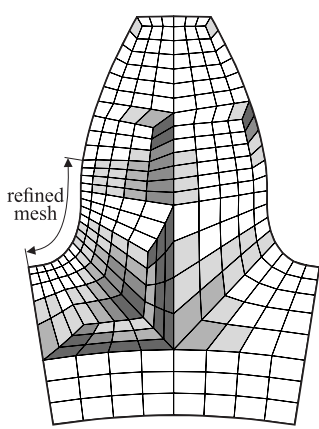

(a) G1FR-PI

285 elem. $/ 325$ nodes $f_{O \text {, max }}=42.2 / f_{\text {o,avg }}=3.4$

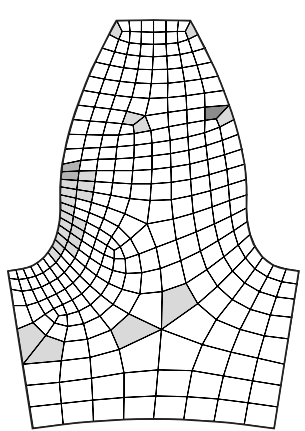

(b) G1FR-DI 285 elem. / 325 nodes $f_{o, \text { max }}=8.0 / f_{0, \text { avg }}=1.1$

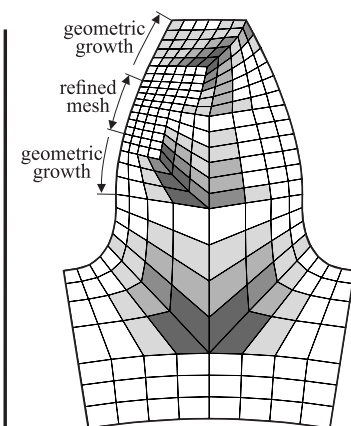

(c) G1PR-PI

262 elem. / 301 nodes

$f_{o, \max }=17.7 / f_{\text {o,avg }}=2.9$

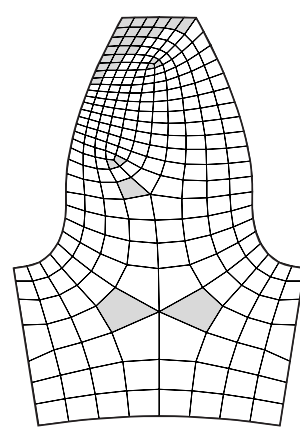

(d) G1PR-DI $f=4.7 / f$

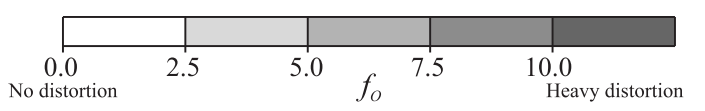

Figure 13: Meshes obtained for cases of study G1FR and G1PR.

study G1FR and G1PR are meshed using the double-improved meshing procedure (G1FR-DI and G1PR-DI, respectively). As expected, the application of the a posteriori mesh quality enhancement technique brings a notable reduction of the average and maximum distortion of the meshes, as well as of the number of heavily distorted elements in the mesh.

Yet, it is known that the application of the a posteriori mesh quality enhancement technique implies an increase of the computational time associated to the meshing procedure. However, this increase is, in general, very small compared to the accuracy improvement and the reduction of the computer time associated to the solution of the finite element model that can be achieved when local mesh refinement is applied.

\subsection{Comparison with Argyris meshing procedure}

Finally, it is interesting to evaluate the performance of the proposed pre-improved meshing procedure compared to the performance of Argyris' procedure. For such a purpose, the cases of study G1FM, G1CM, G2FM and G2CM have been meshed using both procedures, and the resulting meshes are compared in terms of their maximum and average distortion.

To make this comparison more sound, minor modifications have been made in the boundary discretization when applying the Argyris' procedure, in such a way that the resulting meshes have a similar number of nodes and elements than the meshes resulting from the proposed preimproved procedure. Furthermore, distances $d_{1}$ and $d_{2}$ used in the Argyris' procedure (see Fig. 3) have been optimized for each case of study, in order to reduce the average distortion of the mesh by minimizing the average value of Oddy's metric.

Figure 14 shows the meshes obtained when the selected cases of study are meshed using the Argyris' procedure (which are denoted by an A letter). In general, it can be observed that this procedure tends to produce heavily distorted elements in the central part of the tooth section. Besides that, heavily distorted elements also appear at the tip of the tooth and the contact profiles when applying the Argyris meshing procedure to gear geometry G2.

The comparison between the meshes obtained from both procedures shows that the preimproved meshing procedure leads to less distorted meshes than Argyris' procedure, both in 


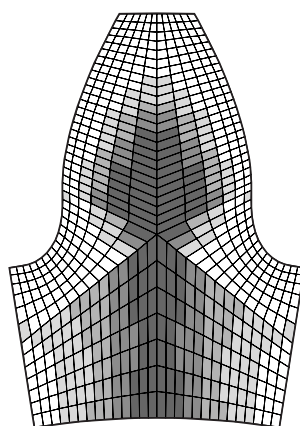

(a) G1FM-A 652 elem. $/ 724$ nodes $f_{O, \text { max }}=26.4 / f_{O, \text { avg }}=3.8$

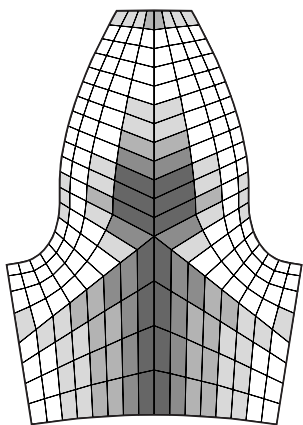

(b) G1CM-A 224 elem. / 265 nodes $f_{O, \max }=13.9 / f_{O, \text { avg }}=3.1$

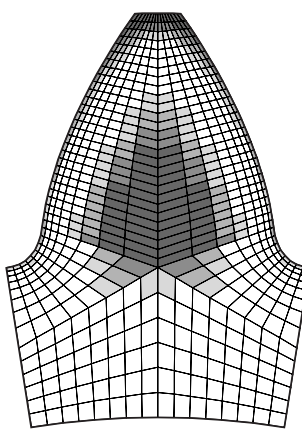

(c) G2FM-A

672 elem. / 739 nodes $f_{\text {o, } \max }=33.0 / f_{\text {o,avg }}=4.0$

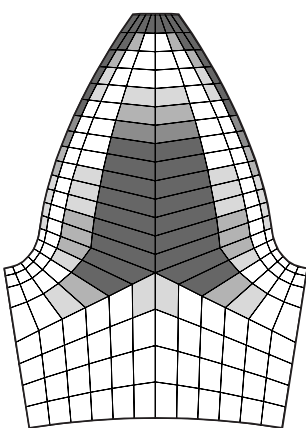

(d) G2CM-A 208 elem. $/ 247$ nodes $f_{o, \text { max }}=33.2 / f_{O, \text { avg }}=5.3$

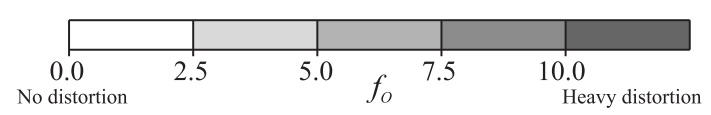

Figure 14: Meshes obtained for cases of study G1FM, G1CM, G2FM and G2CM using Argyris' procedure

terms of average and maximum distortion. A more detailed comparison between the distortion of the meshes produced by both meshing procedures is presented in Fig. 15. In this figure five ranges of Oddy's metric are defined and the percentage of elements in the mesh within each range is shown. It can be observed that in all the studied cases the proposed pre-improved procedure produces a larger percentage of undistorted elements than Argyris' procedure. In the worst of the cases (G2FM-PI), the proposed pre-improved procedure produces $74 \%$ of undistorted elements whereas in the best of the cases (G2FM-A) Argyris' procedure is only able to produce $60 \%$ of elements within this range.

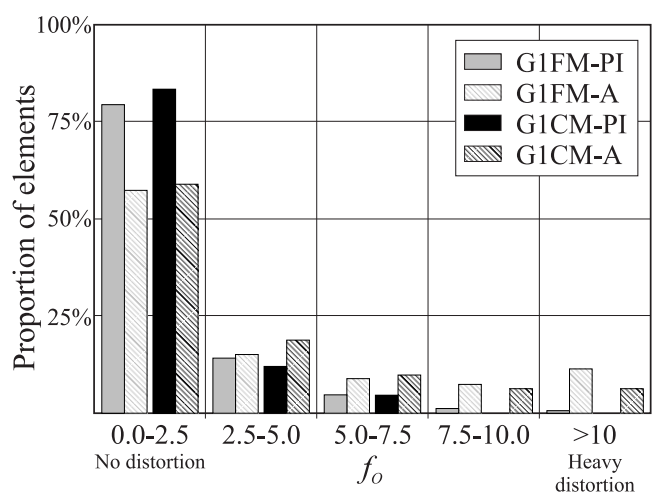

(a) Gear geometry G1

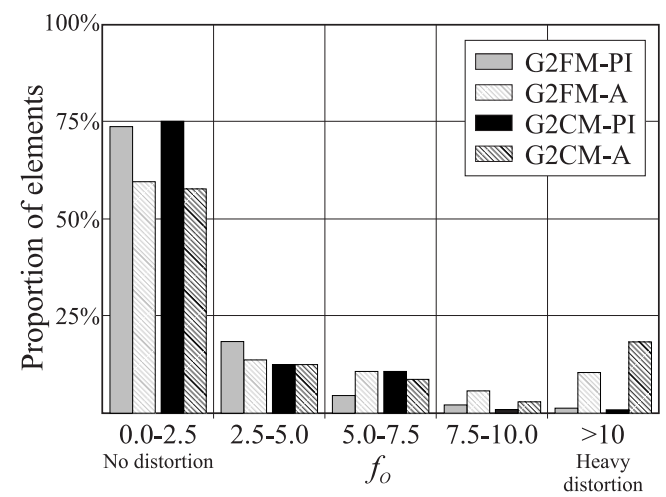

(b) Gear geometry G2

Figure 15: Comparison with Argyris mesh strategy

In contrast, when observing the percentage of heavily distorted elements, in the worst of the cases (G2FM-PI) the proposed pre-improved procedure only produces $1 \%$ of heavily distorted elements, whereas in the best of the cases (G1CM-A) Argyris' procedure produces $6 \%$ of the elements within this range. This means that even in the worst of the cases, the meshes obtained 
using the pre-improved procedure are better (in terms of element distortion) than the meshes obtained using Argyris' procedure. These results evidence the advantages of using the proposed pre-improved meshing procedure compared to the meshing procedure proposed by Argyris.

\section{Conclusions}

In this work, a new procedure for automated generation of two-dimensional quadrilateral meshes of gear drives is proposed. The proposed procedure is based on a multiblock scheme, in which the gear tooth is decomposed into simple patches that are individually meshed using a local meshing scheme. Each of the typical steps of the multiblock schemes has been investigated in depth and, as a result, a fast and simple way to mesh planar sections of gear teeth has been obtained. The performance of the proposed procedure has been illustrated with several numerical examples, which demonstrate the ability of the proposed procedure to mesh different gear geometries under several meshing boundary conditions.

The proposed procedure has been completed with two different mesh quality enhancement techniques, which are aimed to reduce the distortion of the elements in which the gear geometry is discretized. The first one of them is an a priori mesh quality enhancement technique, which is applied before the mesh is generated, and the second one is an a posteriori mesh quality enhancement technique, which is applied after the mesh is generated. The numerical examples have revealed that the proposed a priori mesh quality enhancement technique is capable of reducing the distortion of the resulting meshes without increasing the computational time of the meshing process. In addition, it has been shown that the application of the a posteriori mesh quality enhancement technique can bring further reductions of the distortion of the elements of the mesh.

The performance of the proposed meshing procedure has been compared to the performance of a traditional meshing procedure and, as a result of this comparison, it is observed that the proposed procedure provides meshes with better quality than the traditional approaches. In addition, the proposed meshing procedure covers an important lack of the traditional meshing procedures, which is the performance of local mesh refinement. Examples of local mesh refinement at the contact profile and the fillet of the gear tooth have been shown, demonstrating the capability of the proposed procedure to perform local mesh refinement in different parts of the gear tooth.

In summary, the proposed meshing procedure has the advantage of being simple and provides two important developments regarding the existing procedures to mesh gear drives: the possibility of performing local mesh refinement and a significant improvement in the final quality of the mesh.

\section{Bibliography}

[1] M. Hotait, A. Kahraman, T. Nishino, An investigation of root stresses of hypoid gears with misalignments, Journal of Mechanical Design, Transactions of the ASME 133 (7).

[2] C. Hasl, H. Liu, P. Oster, T. Tobie, K. Stahl, F. fuer Zahnraeder und Getriebebau (Gear Research Centre), Method for calculating the tooth root stress of plastic spur gears meshing with steel gears under consideration of deflectioninduced load sharing, Mechanism and Machine Theory 111 (2017) 152-163.

[3] K. Mao, Gear tooth contact analysis and its application in the reduction of fatigue wear, Wear 262 (11-12) (2007) 1281-1288.

[4] I. Gonzalez-Perez, V. Roda-Casanova, A. Fuentes, F. Sanchez-Marin, J. Iserte, A finite element model for consideration of the torsional effect on the bearing contact of gear drives, Journal of Mechanical Design, Transactions of the ASME 134 (7). 
[5] S. Patil, S. Karuppanan, I. Atanasovska, A. Wahab, Contact stress analysis of helical gear pairs, including frictional coefficients, International Journal of Mechanical Sciences 85 (2014) 205-211.

[6] V. Roda-Casanova, F. Sanchez-Marin, A 2d finite element based approach to predict the temperature field in polymer spur gear transmissions, Mechanism and Machine Theory 133 (2019) 195-210.

[7] C. Fernandes, D. Rocha, R. Martins, L. Magalh£es, J. Seabra, Finite element method model to predict bulk and flash temperatures on polymer gears, Tribology International 120 (2018) 255-268.

[8] F. Litvin, A. Fuentes, Gear Geometry and Applied Theory, Cambridge University Press, 2004.

[9] S. Barone, L. Borgianni, P. Forte, Evaluation of the effect of misalignment and profile modification in face gear drive by a finite element meshing simulation, Journal of Mechanical Design, Transactions of the ASME 126 (5) (2004) 916-924.

[10] H. Ding, Z. Wan, Y. Zhou, J. Tang, A data-driven programming of the human-computer interactions for modeling a collaborative manufacturing system of hypoid gears by considering both geometric and physical performances, Robotics and Computer-Integrated Manufacturing 51 (2018) 121-138.

[11] F. Karpat, S. Ekwaro-Osire, K. Cavdar, F. Babalik, Dynamic analysis of involute spur gears with asymmetric teeth, International Journal of Mechanical Sciences 50 (12) (2008) 1598-1610.

[12] M. Faggioni, F. Samani, G. Bertacchi, F. Pellicano, Dynamic optimization of spur gears, Mechanism and Machine Theory 46 (4) (2011) 544-557.

[13] J. Argyris, A. Fuentes, F. Litvin, Computerized integrated approach for design and stress analysis of spiral bevel gears, Computer Methods in Applied Mechanics and Engineering 191 (11-12) (2002) 1057-1095.

[14] J. Pedrero, M. Pleguezuelos, M. Arts, J. Antona, Load distribution model along the line of contact for involute external gears, Mechanism and Machine Theory 45 (5) (2010) 780-794.

[15] C. Zanzi, J. Pedrero, Application of modified geometry of face gear drive, Computer Methods in Applied Mechanics and Engineering 194 (27-29) (2005) 3047-3066.

[16] N. Cappellini, T. Tamarozzi, B. Blockmans, J. Fiszer, F. Cosco, W. Desmet, Semi-analytic contact technique in a non-linear parametric model order reduction method for gear simulations, Meccanica 53 (1-2) (2018) 49-75.

[17] P. M. Knupp, Remarks on Mesh Quality, 45th AIAA Aerospace Sciences Meeting and Exhibit (January) (2007) 2 $\& 5$.

[18] I. Gonzalez-Perez, A. Fuentes-Aznar, Implementation of a finite element model for stress analysis of gear drives based on multi-point constraints, Mechanism and Machine Theory 117 (2017) 35-47.

[19] M. Barbieri, A. Zippo, F. Pellicano, Adaptive grid-size finite element modeling of helical gear pairs, Mechanism and Machine Theory 82 (2014) 17-32.

[20] Y. Liu, Y. Zhao, M. Liu, X. Sun, Parameterized High-Precision Finite Element Modelling Method of 3D Helical Gears with Contact Zone Refinement, Shock and Vibration 2019 (2019) 1-17.

[21] I. Gonzalez-Perez, A. Fuentes-Aznar, Implementation of a finite element model for gear stress analysis based on tie-surface constraints and its validation through the hertz's theory, Journal of Mechanical Design, Transactions of the ASME 140 (2)

[22] C. Roarty, N. Grosland, Adaptive meshing technique applied to an orthopaedic finite element contact problem. The Iowa orthopaedic journal 24 (2004) 21-29.

[23] A. Keskin, M. Kober, E. Stelldinger, A. Khhorn, H. Bhm, A. Hornig, A. Langkamp, M. Gude, On the quantification of errors of a pre-processing effort reducing contact meshing approach, in: 53rd AIAA Aerospace Sciences Meeting, 2015, p. 12

[24] P. Frey, P. George, Mesh generation applied to finite elements, Hermes Science, 2000.

[25] S. Owen, A survey of unstructured mesh generation technology, in: Proceedings of the 7th International Meshing Roundtable, 1998, pp. 239-267.

[26] M. Farrashkhalvat, J. Miles, Basic Structured Grid Generation: With an introduction to unstructured grid generation, Elsevier Science, 2003.

[27] S. Park, C. Washam, Drag method as a finite element mesh generation scheme, Computers and Structures 10 (1-2) (1979) 343-346.

[28] A. de Oliveira Miranda, L. Martha, Hierarchical template-based quadrilateral mesh generation, Engineering with Computers 33 (4) (2017) 701-715.

[29] W. J. Gordon, C. A. Hall, Construction of curvilinear co-ordinate systems and applications to mesh generation, International Journal for Numerical Methods in Engineering 7 (4) (1973) 461-477.

[30] P. Knupp, S. Steinberg, Fundamentals of grid generation, CRC Press, 1993.

[31] J. Lopez, M. Brovka, J. Escobar, J. Cascn, R. Montenegro, An optimization based method for the construction of 2d parameterizations for isogeometric analysis with t-splines, SEMA SIMAI Springer Series 5 (2015) 91-112.

[32] T. Zhou, K. Shimada, An angle-based approach to two-dimensional mesh smoothing, in: Proceedings of the Ninth International Meshing Roundtable, 2000, p. 373384

[33] A. Oddy, J. Goldak, M. McDill, M. Bibby, Distortion metric for isoparametric finite elements, Transactions of the Canadian Society for Mechanical Engineering 12 (4) (1988) 213-217. 